\title{
ON A DIFFERENCE EQUATION WITH MIN-MAX RESPONSE
}

\section{GEORGE L. KARAKOSTAS and STEVO STEVIĆ}

\author{
Received 25 February 2004
}

\begin{abstract}
We investigate the global behavior of the (positive) solutions of the difference equation $x_{n+1}=\alpha_{n}+F\left(x_{n}, \ldots, x_{n-k}\right), n=0,1, \ldots$, where $\left(\alpha_{n}\right)$ is a sequence of positive reals and $F$ is a min-max function in the sense introduced here. Our results extend several results obtained in the literature.
\end{abstract}

2000 Mathematics Subject Classification: 39A10.

1. Introduction. Let $k$ be a positive integer and let $\mathbb{R}_{+}$be the set of all positive reals. We give the following definition.

DEFINITION 1.1. A function $F: \mathbb{R}_{+}^{k+1} \rightarrow \mathbb{R}_{+}$is called a min-max function if it satisfies the inequality

$$
\frac{\wedge_{j=1}^{k+1} u_{j}}{\vee_{j=1}^{k+1} u_{j}} \leq F\left(u_{1}, u_{2}, \ldots, u_{k+1}\right) \leq \frac{\vee_{j=1}^{k+1} u_{j}}{\wedge_{j=1}^{k+1} u_{j}},
$$

for all $u_{j}>0, j=1, \ldots, k+1$, where, as usual, the symbol $\vee_{j=1}^{n} u_{j}$ stands for the maximum of the variables $u_{j}, j=1, \ldots, n$, and $\wedge_{j=1}^{n} u_{j}$ stands for their minimum.

In Section 2, we give exact information on the form which a min-max function may have.

Simple examples of min-max functions are

$$
F_{1}\left(u_{1}, u_{2}\right):=\frac{u_{2}}{u_{1}}, \quad F_{2}\left(u_{1}, u_{2}\right):=\frac{u_{1}}{u_{2}}
$$

which appear as the response functions, respectively, in the difference equation

$$
y_{n+1}=\alpha+\frac{y_{n-1}}{y_{n}}
$$

studied in [1] and in the difference equation

$$
y_{n+1}=\alpha+\frac{y_{n}}{y_{n-1}}
$$

studied in [2]. These two equations have completely different behavior; see Remark 3.6. Also in $[13,14]$, the second author considered the closely related equation

$$
x_{n+1}=\alpha_{n}+\frac{x_{n-1}}{x_{n}}
$$


where $\left(\alpha_{n}\right)$ is either a periodic sequence (with period two) or a convergent sequence of nonnegative real numbers.

Motivated by the above-mentioned works, in this paper, we study the behavior of the difference equation

$$
x_{n+1}=\alpha_{n}+F\left(x_{n}, \ldots, x_{n-k}\right), \quad n=0,1, \ldots
$$

where the initial conditions $x_{-k}, \ldots, x_{0}$ are positive real numbers, $\left(\alpha_{n}\right)$ is a sequence of positive real numbers, and $F$ is a min-max function.

Since a min-max function takes the value 1 at the diagonal of the space $\mathbb{R}_{+}^{k+1}$, it follows that in case the sequence $\left(\alpha_{n}\right)$ converges to a certain $\alpha$, the positive real number

$$
K:=\alpha+1
$$

is the unique asymptotic equilibrium of (1.6).

Our purpose here is to discuss the boundedness and persistence of (1.6), as well as the attractivity of the asymptotic equilibrium $\alpha+1$, where $\alpha$ is the limit of $\left(\alpha_{n}\right)$ whenever this exists. This follows immediately by Theorem 3.2, where we show that, if $1<\liminf \alpha_{n} \leq \limsup \alpha_{n}<+\infty$, then any solution $\left(x_{n}\right)$ satisfies the relation

$$
1 \leq \frac{\limsup x_{n}}{\liminf x_{n}} \leq \frac{\limsup \alpha_{n}-1}{\liminf \alpha_{n}-1}
$$

Thus, if the sequence $\left(\alpha_{n}\right)$ converges to some $\alpha(>1)$, then any solution with positive initial values converges to the asymptotic equilibrium $K=\alpha+1$. This generalizes [1, Theorem 5.2] and part of [2, Theorem 1]. For the case $\alpha_{n}=1$, for all $n$ (in Theorem 3.3), we show that any nonoscillatory solution converges to 2, while if $F$ satisfies the additional (sufficient) conditions

$$
\begin{aligned}
& u_{i}<\vee_{j \neq i} u_{j} \Longrightarrow F\left(u_{1}, u_{2}, \ldots, u_{k+1}\right)<\frac{\vee_{j \neq i} u_{j}}{u_{i}}, \\
& u_{i}>\wedge_{j \neq i} u_{j} \Longrightarrow F\left(u_{1}, u_{2}, \ldots, u_{k+1}\right)>\frac{\wedge_{j \neq i} u_{j}}{u_{i}},
\end{aligned}
$$

then it is shown in Theorem 3.4 that all solutions converge to 2. Comparing this fact with the results in [1], we see that the pair of conditions (1.9)-(1.10) seems also to be necessary. Indeed, these conditions are not satisfied in case of (1.3) and, as it is shown in [1, Theorem 4.1], it has (nontrivial) solutions which are periodic with period 2.

In Theorem 3.5, we show that if $\alpha_{n}=\alpha<1$, for all $n$, then there is a large class of equations of the form (1.6) which have unbounded (positive) solutions. This result extends [1, Theorem 3.1]. In the Section 4, we give two examples of difference equations with min-max response to illustrate our results.

Also the so-called (2,2)-type equation defined in [6] (where about 50 types of difference equations are presented) includes the equation

$$
x_{n+1}=\frac{A_{1} x_{n}+B_{1} x_{n-1}}{A_{2} x_{n}+B_{2} x_{n-1}} .
$$


Under appropriate choice of the parameters, (1.11) can be written as

$$
x_{n+1}=\alpha+\frac{(\beta+\gamma) x_{n-1}}{\beta x_{n}+\gamma x_{n-1}},
$$

which is of the type (1.6). Thus in this paper, we push further the investigation originated in [6] for such a form of (2,2)-type difference equations.

For other closely related results, which mostly deal with difference equations and inequalities whose response is (or it can be transformed into) a min-max function, see, for instance, $[7,8,9,10,11,12,13,14]$ and the references cited therein.

2. On the min-max functions. In this section, we give a characterization of min-max functions. The result is incorporated in the following theorem.

THEOREM 2.1. A function $F: \mathbb{R}_{+}^{k+1} \rightarrow \mathbb{R}_{+}$is a min-max function if and only if there are nonnegative real-valued functions $a_{j}\left(u_{1}, u_{2}, \ldots, u_{k+1}\right), b_{j}\left(u_{1}, u_{2}, \ldots, u_{k+1}\right), j=1,2, \ldots$, $k+1$, such that

$$
\begin{array}{r}
\sum_{j=1}^{k+1} a_{j}\left(u_{1}, u_{2}, \ldots, u_{k+1}\right)=\sum_{j=1}^{k+1} b_{j}\left(u_{1}, u_{2}, \ldots, u_{k+1}\right)=1, \\
F\left(u_{1}, u_{2}, \ldots, u_{k+1}\right)=\frac{\sum_{j=1}^{k+1} a_{j}\left(u_{1}, u_{2}, \ldots, u_{k+1}\right) u_{j}}{\sum_{j=1}^{k+1} b_{j}\left(u_{1}, u_{2}, \ldots, u_{k+1}\right) u_{j}}
\end{array}
$$

for all $\left(u_{1}, u_{2}, \ldots, u_{k+1}\right) \in \mathbb{R}_{+}^{k+1}$.

Proof. The "if" part is easily proved by using the form of $F$ and the conditions on the coefficients $a_{j}, b_{j}$.

To show the inverse, assume that $F\left(u_{1}, u_{2}, \ldots, u_{k+1}\right)$ is a min-max function and fix any element $\left(u_{1}, u_{2}, \ldots, u_{k+1}\right) \in \mathbb{R}_{+}^{k+1}$. We let

$$
v:=\wedge_{j=1}^{k+1} u_{j}, \quad w:=\vee_{j=1}^{k+1} u_{j},
$$

thus $v=u_{j_{1}}$ and $w=u_{j_{2}}$, for two indices $j_{1}, j_{2} \in\{1,2, \ldots, k+1\}$.

From the definition of the min-max functions, we know that the value $F\left(u_{1}\right.$, $\left.u_{2}, \ldots, u_{k+1}\right)$ lies in the interval $[v / w, w / v]$, thus there is a number $a \in[0,1]$ such that

$$
F\left(u_{1}, u_{2}, \ldots, u_{k+1}\right)=a \frac{w}{v}+(1-a) \frac{v}{w}
$$

Let

$$
b:=\frac{(1-a) v^{2}}{a w^{2}+(1-a) v^{2}} .
$$

It is clear that $b$ belongs to the interval $[0,1]$, and it depends on $v, w$ (thus on $\left.u_{1}, u_{2}, \ldots, u_{k+1}\right)$. By some simple calculations, we obtain

$$
(b w+(1-b) v)\left(a \frac{w}{v}+(1-a) \frac{v}{w}\right)=a w+(1-a) v
$$


and consequently we get

$$
F\left(u_{1}, u_{2}, \ldots, u_{k+1}\right)=a \frac{w}{v}+(1-a) \frac{v}{w}=\frac{a w+(1-a) v}{b w+(1-b) v}
$$

This proves the theorem since we can set $a_{j}\left(u_{1}, u_{2}, \ldots, u_{k+1}\right):=0$, if $j \neq j_{1}, j_{2}$, while $a_{j_{1}}\left(u_{1}, u_{2}, \ldots, u_{k+1}\right)=1-a$ and $a_{j_{2}}\left(u_{1}, u_{2}, \ldots, u_{k+1}\right)=a$. Similar substitutions are used for the denominator. The proof is complete.

REMARK 2.2. The quotient of any two elements of the class of all $f: \mathbb{R}_{+}^{k+1} \rightarrow \mathbb{R}_{+}$ which satisfy an inequality of the form

$$
\wedge_{j=1}^{k+1} u_{j} \leq f\left(u_{1}, u_{2}, \ldots, u_{k+1}\right) \leq \vee_{j=1}^{k+1} u_{j}
$$

produces a min-max function.

3. The main results. Our first result refers to the boundedness of the solutions.

THEOREM 3.1. Consider (1.6), where $F$ is a min-max function and the sequence $\left(\alpha_{n}\right)$ satisfies

$$
1<C:=\inf \alpha_{n} \leq \sup \alpha_{n}=: B<+\infty
$$

Then any solution $\left(x_{n}\right)$ with positive initial values satisfies the condition

$$
\min \left\{\wedge_{j=1}^{k+1} x_{j}, \frac{L C}{L-1}\right\} \leq x_{n} \leq L,
$$

for all $n=1,2, \ldots$, where

$$
L:=\max \left\{\vee_{j=1}^{k+1} x_{j}, \frac{B C}{C-1}\right\}
$$

Also, if $\alpha_{n}=\alpha=1$, for all $n$, then it holds that

$$
M \leq x_{n} \leq \frac{M}{M-1}
$$

for all $n \geq 1$, where

$$
M:=\min \left\{\wedge_{j=1}^{k+1} x_{j}, \frac{\vee_{j=1}^{k+1} x_{j}}{\vee_{j=1}^{k+1} x_{j}-1}\right\} .
$$

Proof. Let $n>k+1$. From (1.6), for all $j \geq 1$, we have

$$
C<x_{j} \leq \vee_{i=1}^{n} x_{i}
$$


Also, for all $j=k+2, k+3, \ldots, n$, we get

$$
x_{j} \leq B+\frac{\vee_{i=j-k-1}^{j-1} x_{i}}{C} \leq B+\frac{\vee_{i=1}^{n} x_{i}}{C}
$$

These facts imply that

$$
\vee_{j=1}^{n} x_{j} \leq \max \left\{\vee_{i=1}^{k+1} x_{i}, B+\frac{\vee_{i=1}^{n} x_{i}}{C}\right\}
$$

from which we get

$$
x_{n} \leq \vee_{i=1}^{n} x_{i} \leq \max \left\{\vee_{i=1}^{k+1} x_{i}, \frac{B C}{C-1}\right\}
$$

and therefore,

$$
C<x_{m} \leq L
$$

for all $m=1,2, \ldots$.

Next let $n>k+1$. From (3.10) and (1.6), it follows that for all $j=k+2, k+3, \ldots, n$, it holds that

$$
x_{j} \geq C+\frac{\wedge_{i=j-k-1}^{j-1} x_{i}}{L} \geq C+\frac{\wedge_{i=1}^{n} x_{i}}{L} .
$$

Therefore, we have

$$
x_{j} \geq \min \left\{\wedge_{i=1}^{k+1} x_{i}, C+\frac{\wedge_{i=1}^{n} x_{i}}{L}\right\},
$$

for all $j=1,2, \ldots$. This implies that

$$
\wedge_{i=1}^{n} x_{i} \geq \min \left\{\wedge_{i=1}^{k+1} x_{i}, C+\frac{\wedge_{i=1}^{n} x_{i}}{L}\right\}
$$

and so

$$
\wedge_{i=1}^{n} x_{i} \geq \min \left\{\wedge_{i=1}^{k+1} x_{i}, \frac{L C}{L-1}\right\} .
$$

This gives

$$
x_{n} \geq \wedge_{i=1}^{n} x_{i} \geq \min \left\{\wedge_{i=1}^{k+1} x_{i}, \frac{L C}{L-1}\right\},
$$

which, together with (3.10), proves the first result of the theorem. 
Next assume that $\alpha_{n}=1, n=0,1, \ldots$. To show inequality (3.4), we observe that

$$
M \leq x_{n} \leq \frac{M}{M-1}
$$

for all $n=1,2, \ldots, k+1$. Also from (1.6), we get

$$
\begin{gathered}
x_{k+2} \geq 1+\frac{\wedge_{j=1}^{k+1} x_{j}}{\vee_{j=1}^{k+1} x_{j}} \geq 1+\frac{M}{M /(M-1)}=M, \\
x_{k+2} \leq 1+\frac{\vee_{j=1}^{k+1} x_{j}}{\wedge_{j=1}^{k+1} x_{j}} \leq 1+\frac{M /(M-1)}{M}=\frac{M}{M-1} .
\end{gathered}
$$

These arguments and the induction complete the proof.

THEOREM 3.2. Consider (1.6), where $F$ is a continuous min-max function and the sequence $\left(\alpha_{n}\right)$ satisfies the condition

$$
1<\liminf \alpha_{n} \leq \limsup \alpha_{n}<+\infty
$$

Then any (positive) solution $\left(x_{n}\right)$ satisfies relation (1.8). Hence, if the sequence $\left(\alpha_{n}\right)$ converges to some $\alpha(>1)$, then $\left(x_{n}\right)$ converges to (a constant, which, therefore, is equal to) $\alpha+1=: K$.

Proof. Let $\left(x_{n}\right)$ be a solution. From Theorem 3.1, the solution is bounded, thus there are two-sided sequences, $\left(y_{m}\right)$ (upper full limiting sequence) and $\left(z_{m}\right)$ (lower full limiting sequence) of $\left(x_{n}\right)$ (see, e.g., [3, 4, 5]), satisfying (1.6), for all integers $m$, and such that

$$
\liminf x_{n}=z_{0} \leq z_{m}, \quad y_{m} \leq y_{0}=\lim \sup x_{n},
$$

for all $m$. Let $a_{0}:=\liminf \alpha_{n}$ and $a^{0}:=\lim \sup \alpha_{n}$. Then from (1.6), we have

$$
y_{0} \leq a^{0}+\frac{y_{0}}{z_{0}}, \quad z_{0} \geq a_{0}+\frac{z_{0}}{y_{0}}
$$

Combining these two relations, we obtain (1.8).

THEOREM 3.3. Consider (1.6), where $\alpha_{n}=1, n=0,1, \ldots$, and $F$ is a min-max function. Then every nonoscillatory (positive) solution converges to the equilibrium $K=2$.

Proof. Assume first that $x_{n} \geq 2$, for all $n \geq-k$. Set $u_{n}:=x_{n}-2$. From Theorem 2.1, we know that $F$ may take the form (2.1), where the (nonnegative) functions $a_{j}, b_{j}$ satisfy

$$
\sum_{j=1}^{k+1} a_{j}\left(x_{n}, \ldots, x_{n-k}\right)=\sum_{j=1}^{k+1} b_{j}\left(x_{n}, \ldots, x_{n-k}\right)=1 .
$$


Then we obtain

$$
\begin{aligned}
u_{n+1} & =\frac{\sum_{j=1}^{k+1} a_{j}\left(x_{n}, \ldots, x_{n-k}\right) u_{n+1-j}}{\sum_{j=1}^{k+1} b_{j}\left(x_{n}, \ldots, x_{n-k}\right) x_{n+1-j}}-\frac{\sum_{j=1}^{k+1} b_{j}\left(x_{n}, \ldots, x_{n-k}\right) u_{n+1-j}}{\sum_{j=1}^{k+1} b_{j}\left(x_{n}, \ldots, x_{n-k}\right) x_{n+1-j}} \\
& \leq \frac{\sum_{j=1}^{k+1} a_{j}\left(x_{n}, \ldots, x_{n-k}\right) u_{n+1-j}}{\sum_{j=1}^{k+1} b_{j}\left(x_{n}, \ldots, x_{n-k}\right) x_{n+1-j}} \leq \frac{1}{2} \vee_{n-k}^{n} u_{j} .
\end{aligned}
$$

Our intention is to show that $\lim u_{n}=0$. To this end, we can either use [7, Lemma 1] or proceed as follows.

Let $\left(Y_{m}\right)$ be an upper full limiting sequence of $\left(u_{n}\right)$ with $Y_{m} \leq Y_{0}=\lim \sup u_{n}$, for all integers $m$. Then, from the previous arguments, it follows that it satisfies the inequality

$$
Y_{0} \leq \frac{1}{2} Y_{0}
$$

thus we have $Y_{0}=0$. This and the fact that $u_{n} \geq 0$ imply that $\lim x_{n}=2$.

Next, assume that $x_{n} \leq 2$, for all $n \geq-k$. Set $v_{n}:=2-x_{n}$. From (1.3) and by using the form of the function $F$, we obtain

$$
\begin{aligned}
v_{n+1} & =\frac{\sum_{j=1}^{k+1} a_{j}\left(x_{n}, \ldots, x_{n-k}\right) v_{n+1-j}}{\sum_{j=1}^{k+1} b_{j}\left(x_{n}, \ldots, x_{n-k}\right) x_{n+1-j}}-\frac{\sum_{j=1}^{k+1} b_{j}\left(x_{n}, \ldots, x_{n-k}\right) v_{n+1-j}}{\sum_{j=1}^{k+1} b_{j}\left(x_{n}, \ldots, x_{n-k}\right) x_{n+1-j}}, \\
& \leq \frac{\sum_{j=1}^{k+1} a_{j}\left(x_{n}, \ldots, x_{n-k}\right) v_{n+1-j}}{\sum_{j=1}^{k+1} b_{j}\left(x_{n}, \ldots, x_{n-k}\right) x_{n+1-j}} \leq \frac{1}{M} \vee_{j=n-k}^{n} v_{j},
\end{aligned}
$$

where $M(>1)$ is the number defined in Theorem 3.1. By using this fact and following the same procedure as in the first case, we derive that $\lim _{n \rightarrow \infty} v_{n}=0$, which implies that $\lim x_{n}=2$, as desired.

THEOREM 3.4. Consider (1.6), where $\alpha_{n}=1, n=0,1, \ldots$, and $F$ is a continuous minmax function satisfying the properties (1.9) and (1.10). Then every (positive) solution converges to the equilibrium $K=2$.

Proof. Let $\left(x_{n}\right)$ be a solution. Then by Theorem 3.1, $\left(x_{n}\right)$ is bounded. Consider an upper full limiting sequence $\left(y_{m}\right)$ and a lower full limiting sequence $\left(z_{m}\right)$ of $\left(x_{n}\right)$, as above. From (1.6), we have

$$
y_{0} \leq 1+\frac{y_{0}}{z_{0}}, \quad z_{0} \geq 1+\frac{z_{0}}{y_{0}}
$$

and therefore, we get

$$
y_{0} z_{0}=y_{0}+z_{0}
$$

This gives

$$
\frac{1}{y_{0}}+\frac{1}{z_{0}}=1
$$


If it happens that $y_{0}, z_{0}>2$, or $y_{0}, z_{0}<2$, then we should have $1 / y_{0}, 1 / z_{0}<1 / 2$ and $1 / y_{0}, 1 / z_{0}>1 / 2$, respectively. Both these arguments contradict (3.27). Therefore, we must have

$$
z_{0} \leq 2 \leq y_{0}
$$

Assume that there is some $j \in\{-k-1, \ldots,-1\}$ such that $y_{j}<y_{0}$ and let $j_{0}$ be an index such that

$$
y_{j_{0}}=\wedge_{j=-k-1}^{-1} y_{j}
$$

Then from (1.9), we get

$$
y_{j_{0}}<\vee_{j \neq j_{0}} y_{j} \leq y_{0}
$$

and so from (1.6) and condition (1.9), we have

$$
y_{0}=1+F\left(y_{-1}, \ldots, y_{-k-1}\right)<1+\frac{y_{0}}{y_{j_{0}}} \leq 1+\frac{y_{0}}{z_{0}}
$$

This gives $y_{0} z_{0}<y_{0}+z_{0}$, contradicting (3.26). Thus we have $y_{j}=y_{0}$, for all $j=$ $-k-1, \ldots,-1$, and therefore,

$$
y_{0}=1+F\left(y_{-1}, \ldots, y_{-k-1}\right)=1+F\left(y_{0}, \ldots, y_{0}\right)=2
$$

Similarly, we can use condition (1.10) to obtain $z_{0}=2$. The proof is complete.

Our final result refers to the case $\alpha \in[0,1)$. We show that in this case, there are equations of the form (1.3) which admit unbounded solutions.

THEOREM 3.5. Consider the equation

$$
x_{n+1}=\alpha+\frac{\sum_{i=0}^{m} a_{i} x_{n-2 i-1}}{\sum_{i=0}^{m} b_{i} x_{n-2 i}},
$$

where $m \in \mathbb{N}, \alpha \in[0,1)$, and where the coefficients $a_{j}$ and $b_{j}, j=0, \ldots, m$, are nonnegative constants which satisfy the conditions

$$
\sum_{i=0}^{m} a_{i}=\sum_{i=0}^{m} b_{i}
$$

Then there exist unbounded solutions of (3.33).

Proof. Obviously, without loss of the generality, we can assume that $\sum_{i=0}^{m} a_{i}=$ $\sum_{i=0}^{m} b_{i}=1$. 
Assume that $\alpha \in(0,1)$. We choose the initial conditions such that

$$
\begin{gathered}
x_{-(2 m+1)}, \ldots, x_{-1}>\frac{1}{1-\alpha}>1+\alpha, \\
\alpha<x_{-2 m}, \ldots, x_{0}<1 .
\end{gathered}
$$

We set

$$
D:=\wedge_{i=0}^{m} x_{-(2 i+1)}
$$

and observe that

$$
D>\frac{1}{1-\alpha} .
$$

From (3.33), we have

$$
\begin{aligned}
x_{1} & =\alpha+\frac{\sum_{i=0}^{m} a_{i} x_{-(2 i+1)}}{\sum_{i=0}^{m} b_{i} x_{-2 i}}>\alpha+\sum_{i=0}^{m} a_{i} x_{-(2 i+1)}>\alpha+D, \\
x_{2} & =\alpha+\frac{\sum_{i=0}^{m} a_{i} x_{1-(2 i+1)}}{\sum_{i=0}^{m} b_{i} x_{1-2 i}}<\alpha+\frac{1}{\sum_{i=0}^{m} b_{i} x_{1-2 i}} \\
& =\alpha+\frac{1}{b_{0} x_{1}+b_{1} x_{-1}+\cdots+b_{m} x_{-2 m+1}} \leq \alpha+\frac{1}{b_{0}(\alpha+D)+\left(1-b_{0}\right)(1 /(1-\alpha))} \\
& \leq \alpha+\frac{1}{b_{0}(\alpha+1 /(1-\alpha))+\left(1-b_{0}\right)(1 /(1-\alpha))}=\alpha+\frac{1}{b_{0} \alpha+1 /(1-\alpha)} \leq 1, \\
x_{3} & =\alpha+\frac{\sum_{i=0}^{m} a_{i} x_{2-(2 i+1)}}{\sum_{i=0}^{m} b_{i} x_{2-2 i}}>\alpha+\sum_{i=0}^{m} a_{i} x_{2-(2 i+1)} \\
& \geq \alpha+\min \left\{x_{1}, x_{-1}, \ldots, x_{-2 m+1}\right\} \geq \alpha+\min \left\{x_{1}, x_{-1}, \ldots, x_{-2 m-1}\right\} \\
& =\alpha+\min \left\{x_{1}, \min \left\{x_{-1}, \ldots, x_{-2 m-1}\right\}\right\} \geq \alpha+D .
\end{aligned}
$$

Following the same procedure, we get

$$
x_{2 j+1}>\alpha+D, \quad x_{2 j+2}<1,
$$

for all $j=0,1, \ldots, m$. By induction, we obtain

$$
x_{(2 m+2) j-(2 s+1)}>\alpha j+D,
$$

for all $j \in \mathbb{N}$ and $s=0,1, \ldots, m$, as well as

$$
\alpha<x_{2 n}<1, \quad n=-m,-(m-1), \ldots,-1, \ldots .
$$

Inequality (3.40) implies the desired result in case $\alpha>0$.

Assume that $\alpha=0$. Choose $\varepsilon \in(0,1)$ and the initial conditions such that

$$
\begin{gathered}
x_{-(2 m+1)}, \ldots, x_{-1}>\frac{1}{1-\varepsilon}, \\
0<x_{-2 m}, \ldots, x_{0}<1-\varepsilon .
\end{gathered}
$$


From (3.33), we have

$$
\begin{aligned}
x_{1} & =\frac{\sum_{i=0}^{m} a_{i} x_{-(2 i+1)}}{\sum_{i=0}^{m} b_{i} x_{-2 i}}>\frac{1 /(1-\varepsilon)}{1-\varepsilon}=\frac{1}{(1-\varepsilon)^{2}}>\frac{1}{1-\varepsilon}, \\
x_{2} & =\frac{\sum_{i=0}^{m} a_{i} x_{1-(2 i+1)}}{\sum_{i=0}^{m} b_{i} x_{1-2 i}}<\frac{1-\varepsilon}{b_{0} x_{1}+\left(1-b_{0}\right)(1 /(1-\varepsilon))} \\
& \leq \frac{1-\varepsilon}{b_{0}\left(1 /(1-\varepsilon)^{2}\right)+\left(1-b_{0}\right)(1 /(1-\varepsilon))}<1-\varepsilon .
\end{aligned}
$$

Following the same procedure, we get

$$
\begin{gathered}
x_{2 j+1}>\frac{1}{(1-\varepsilon)^{2}}>\frac{1}{1-\varepsilon}, \\
x_{2 j+2}<1-\varepsilon,
\end{gathered}
$$

for all $j=0,1, \ldots, m$. By induction, we obtain

$$
x_{(2 m+2) j-(2 s+1)}>\frac{1}{(1-\varepsilon)^{j+1}},
$$

for all $j \in \mathbb{N}$ and $s=0,1, \ldots, m$, as well as

$$
0<x_{2 n}<1-\varepsilon, \quad n=1,2, \ldots
$$

From (3.45), the result follows.

REMARK 3.6. Equation (3.33) includes the special case (1.3). Thus for $\alpha \in(0,1)$, Theorem 3.5 applies and therefore, (1.3) has unbounded solutions with positive initial values. On the other hand, (3.33) does not include the case (1.4) and as proved in [2], for the same values of $\alpha,(1.4)$ has a global attractor.

REMARK 3.7. By some modifications of the proof of Theorem 3.5, we can prove the following result.

THEOREM 3.8. Consider the equation

$$
x_{n+1}=\alpha_{n}+\frac{\sum_{i=0}^{m} a_{i} x_{n-2 i-1}}{\sum_{i=0}^{m} b_{i} x_{n-2 i}},
$$

where $m \in \mathbb{N},\left(\alpha_{n}\right)$ is a sequence of positive real numbers such that $\lim _{n \rightarrow \infty} \alpha_{n}=: A \in$ $[0,1)$, and where the coefficients $a_{j}$ and $b_{j}, j=0, \ldots, m$, are nonnegative constants which satisfy the conditions

$$
\sum_{i=0}^{m} a_{i}=\sum_{i=0}^{m} b_{i}
$$

Then there exist unbounded solutions of (3.47). 


\section{Some illustrative examples}

EXAMPLE 4.1. Consider the difference equation

$$
x_{n+1}=\alpha+\frac{\beta x_{n}+\gamma x_{n}^{2}+\delta x_{n-1}^{2}}{\beta x_{n}+\gamma x_{n} x_{n-1}+\delta x_{n-1}^{2}},
$$

where all the coefficients are positive real numbers. The rational function on the righthand side is a min-max function, since it can be written in the form

$$
\frac{\left(\left(\beta+\gamma x_{n}\right) /\left(\beta+\gamma x_{n}+\delta x_{n-1}\right)\right) x_{n}+\left(\delta x_{n-1} /\left(\beta+\gamma x_{n}+\delta x_{n-1}\right)\right) x_{n-1}}{\left(\beta /\left(\beta+\gamma x_{n}+\delta x_{n-1}\right)\right) x_{n}+\left(\left(\gamma x_{n}+\delta x_{n-1}\right) /\left(\beta+\gamma x_{n}+\delta x_{n-1}\right)\right) x_{n-1}} .
$$

Thus, from Theorems 3.2 and 3.4, we conclude that, for every fixed $\alpha \geq 1$, any solution of (4.1) converges to the equilibrium $\alpha+1$. Notice that conditions (1.9) and (1.10) are also satisfied.

EXAMPLE 4.2. Consider the difference equation

$$
x_{n+1}=\alpha+\frac{\sum_{j_{i} \in\{n, n-1, n-2\}} x_{j_{1}} x_{j_{2}} x_{j_{3}}}{x_{n}^{3}+x_{n-1}^{3}+x_{n-2}^{3}+6 x_{n} x_{n-1} x_{n-2}},
$$

where $\alpha \geq 0$. This is a third-order difference equation whose response on the right-hand side is a min-max function. Indeed, this can be written in the form

$$
\frac{\sum_{j_{i} \in\{n, n-1, n-2\}, j_{1} \neq j_{2} \neq j_{3} \neq j_{1}}\left(\left(x_{j_{1}}^{2}+x_{j_{1}} x_{j_{2}}+x_{j_{1}} x_{j_{3}}\right) /\left(x_{n}+x_{n-1}+x_{n-2}\right)^{2}\right) x_{j_{1}}}{\sum_{j_{i} \in\{n, n-1, n-2\}, j_{1} \neq j_{2} \neq j_{3} \neq j_{1}}\left(\left(x_{j_{1}}^{2}+2 x_{j_{2}} x_{j_{3}}\right) /\left(x_{n}+x_{n-1}+x_{n-2}\right)^{2}\right) x_{j_{1}}} .
$$

Here, again, Theorems 3.2 and 3.4 apply and we conclude that in case $\alpha \geq 1$, any solution of (4.3) converges to $\alpha+1$.

\section{REFERENCES}

[1] A. M. Amleh, E. A. Grove, G. Ladas, and D. A. Georgiou, On the recursive sequence $x_{n+1}=$ $\alpha+x_{n-1} / x_{n}$, J. Math. Anal. Appl. 233 (1999), no. 2, 790-798.

[2] R. DeVault, G. Ladas, and S. W. Schultz, On the recursive sequence $x_{n+1}=A / x_{n}+1 / x_{n-2}$, Proc. Amer. Math. Soc. 126 (1998), no. 11, 3257-3261.

[3] G. L. Karakostas, A discrete semi-flow in the space of sequences and study of convergence of sequences defined by difference equations, M. E. Greek Math. Soc. 30 (1989), 66-74.

[4] G. L. Karakostas, Ch. G. Philos, and Y. G. Sficas, The dynamics of some discrete population models, Nonlinear Anal. 17 (1991), no. 11, 1069-1084.

[5] G. L. Karakostas and S. Stević, Slowly varying solutions of the difference equation $x_{n+1}=$ $f\left(x_{n}, \ldots, x_{n-k}\right)+g\left(n, x_{n}, x_{n-1}, \ldots, x_{n-k}\right)$, J. Difference Equ. Appl. 10 (2004), no. 3, 249-255.

[6] M. R. S. Kulenović and G. Ladas, Dynamics of Second Order Rational Difference Equations. With Open Problems and Conjectures, Chapman \& Hall/CRC, Florida, 2002.

[7] S. Stević, Behavior of the positive solutions of the generalized Beddington-Holt equation, Panamer. Math. J. 10 (2000), no. 4, 77-85.

[8] - A generalization of the Copson's theorem concerning sequences which satisfy a linear inequality, Indian J. Math. 43 (2001), no. 3, 277-282.

[9] _ A note on bounded sequences satisfying linear inequalities, Indian J. Math. 43 (2001), no. 2, 223-230. 
[10] On the recursive sequence $x_{n+1}=-1 / x_{n}+A / x_{n-1}$, Int. J. Math. Math. Sci. 27 (2001), no. 1, 1-6.

[11] _ A global convergence result, Indian J. Math. 44 (2002), no. 3, 361-368.

[12] _ A global convergence results with applications to periodic solutions, Indian J. Pure Appl. Math. 33 (2002), no. 1, 45-53.

[13] - On the recursive sequence $x_{n+1}=\alpha_{n}+x_{n-1} / x_{n}$, Int. J. Math. Sci. 2 (2003), no. 2, 237-243.

[14] On the recursive sequence $x_{n+1}=\alpha_{n}+x_{n-1} / x_{n}$. II, Dyn. Contin. Discrete Impuls. Syst. Ser. A Math. Anal. 10 (2003), no. 6, 911-916.

George L. Karakostas: Department of Mathematics, University of Ioannina, 45110 Ioannina, Greece

E-mail address: gkarako@cc.uoi.gr

Stevo Stević: Mathematical Institute of the Serbian Academy of Sciences and Arts, Knez Mihailova 35, 11000 Beograd, Serbia

E-mail address: sstevic@ptt.yu; sstevo@matf.bg.ac.yu 


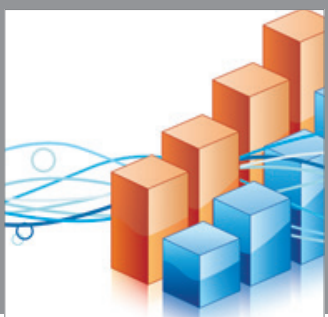

Advances in

Operations Research

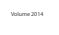

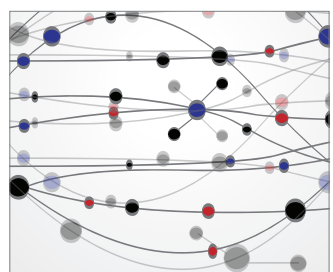

\section{The Scientific} World Journal
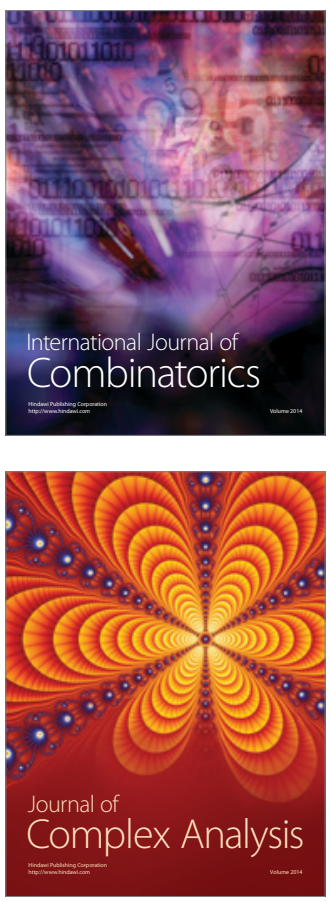

International Journal of

Mathematics and

Mathematical

Sciences
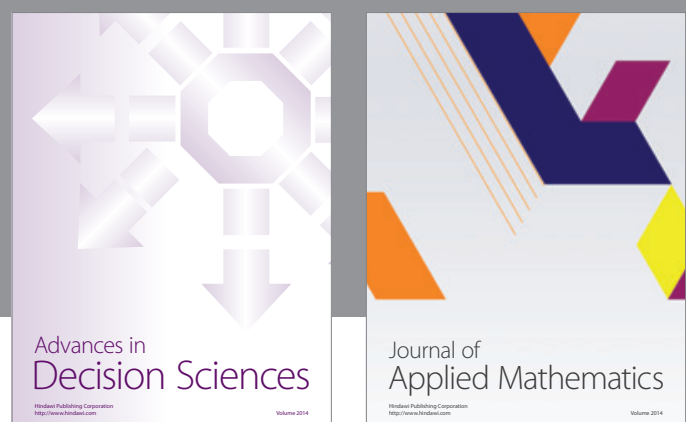

Journal of

Applied Mathematics
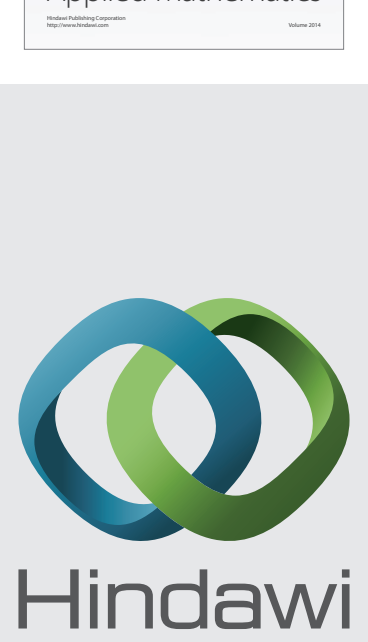

Submit your manuscripts at http://www.hindawi.com
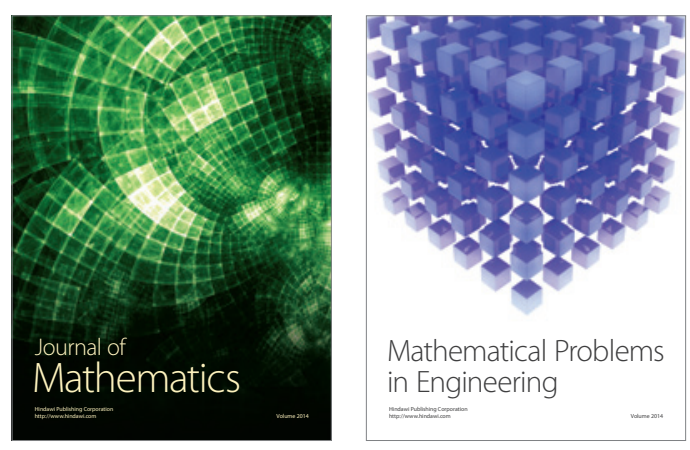

Mathematical Problems in Engineering
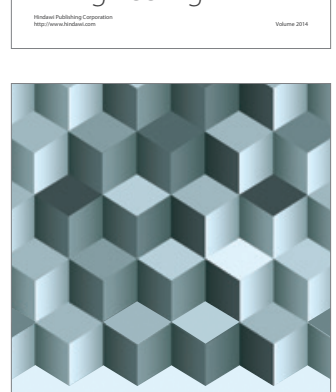

Journal of

Function Spaces
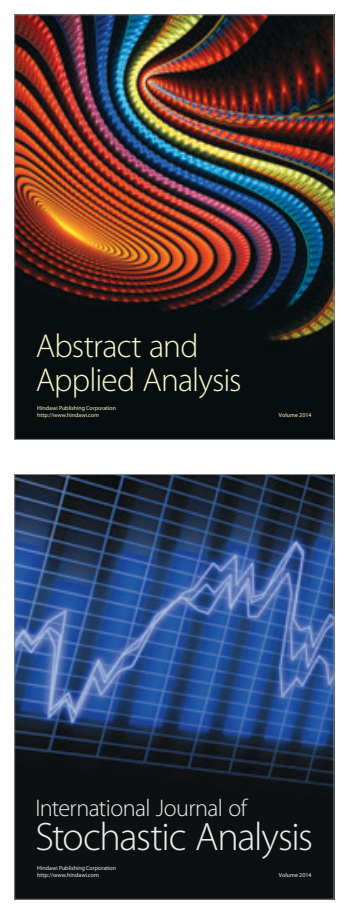

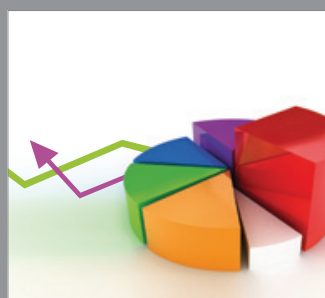

ournal of

Probability and Statistics

Promensencen
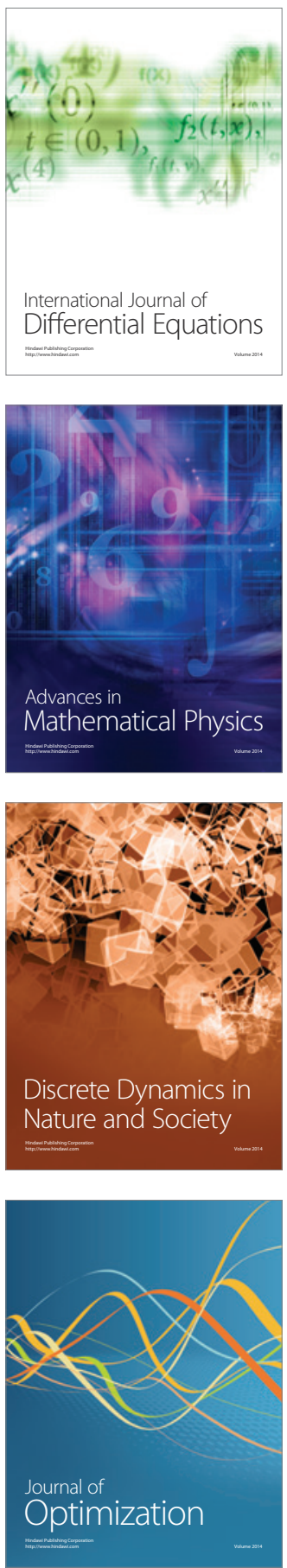